\title{
FINE STRUCTURE OF MASS AND CHARGE DISTRIBUTION IN LOW ENERGY FISSION
}

\author{
H. N. ERTEN, ${ }^{*}$ N. K. ARAS ${ }^{* *}$ \\ *Bilkent University, Ankara (Turkey) \\ **Middle East Technical University, Ankara (Turkey)
}

(Received April 18, 1991)

\begin{abstract}
Studies of finer details in mass and charge distribution in fission leads to a better understanding of the fission process. Experimental determination of independent and cumulative yields using radiochemical techniques as well as mass spectrometers and fission product recoil separators form the basis of such studies. It has been established that closed shells as well as an even number of nucleons influence both mass and charge distributions. The magnitudes of these effects may be estimated from existing experimental yield data and various fission models. Using our measurements of several fission yields and those existing in the literature we have calculated even-odd proton and neutron effects for various low energy fissioning sy stems. Where enough data existed, direct calculations were made, whereas for other cases the $Z_{p}$-model of WAHL has been used. It is found that the even-odd proton effect is well established and pronounced in thermal neutron fission of ${ }^{235} \mathrm{U}$ and ${ }^{233} \mathrm{U}$. Lesser effects were found for reactor neutron induced fission of ${ }^{23}{ }^{2} \mathrm{Th}$, thermal neutron fission of ${ }^{239} \mathrm{Pu}$ and spontaneous fission of ${ }^{245} \mathrm{Cm}$ and ${ }^{249} \mathrm{Cf}$. No effect seems to exist in the thermal neutron fission of ${ }^{241} \mathrm{Pu}$ and the spontaneous fission of ${ }^{252} \mathrm{Cf}$. The even-odd neutron effect is found to be much lower than the corresponding proton effect in ${ }^{235} \mathrm{U}$ and ${ }^{233} \mathrm{U}$ fissions and is nonexistent in the rest of the fissioning systems.
\end{abstract}

\section{Introduction}

Mass, charge and kinetic energy distributions in fission have been the subject of extensive experimental and theoretical studies since the discovery of fission fifty years ago. ${ }^{1}$ Radiochemical methods have been primarily used for fission yield measurements supplemented by mass-spectrometric and fission product recoil techniques. These measurements, particularly those of the independent yields are difficult. As a result, an almost complete set of experimental independent yield data exists for the thermal neutron fission of ${ }^{235} \mathrm{U}$ only followed by ${ }^{233} \mathrm{U}$ and ${ }^{239} \mathrm{Pu}$ fissions. For the spontaneous fission of ${ }^{252} \mathrm{Cf}^{f}$ only about $35 \%$ of the independent yields has been measured. On the other hand, mass distribution, which is characterized by chain yield measurements, has been much better established than charge distribution in low 
energy fission. ${ }^{2},{ }^{3}$ Recently all available yield data in low eneroy fission were compiled by WAHL. ${ }^{2}$

We have also been measuring fission yields of several products in some low energy fissioning systems over the years. ${ }^{4-9}$ The results of such studies together with recent compilations ${ }^{2}, 10$ have been used in reevaluating fine structure effects on the mass and charge distributions in low energy fission. AMIEL and FELDSTEIN ${ }^{11}$ were the first to carry out a systematic study of the even-odd effect in the thermal neutron fission of ${ }^{235} \mathrm{U}$ and ${ }^{233} \mathrm{U}$. This effect was further explored by many investigators. ${ }^{2}, 10,12-14$

\section{Calculations}

Average masses of light peak fission products have been calculated from the relation:

$$
\mathrm{A}_{\mathrm{L}}=\frac{\sum_{\text {all }}^{\mathrm{A}_{\mathrm{L}}} \mathrm{CY}(\mathrm{A}) \cdot \mathrm{A}_{\mathrm{L}}}{\sum_{\text {all }}^{\mathrm{A}_{\mathrm{L}}} \mathrm{CY}(\mathrm{A})}
$$

where CY(A) - yield of mass number A chain,

$A_{L} \quad-$ mass number of light products.

A similar expression was used for the heavy peak $A_{H}$ calculation. The even-odd effect, defined as the enhancement of independent fission yields of even $\mathrm{Z}$ and even $\mathrm{N}$ products relative to odd ones can be determiner using the relation:

$$
\mathrm{EOZ}=\frac{\sum_{\text {all }}^{\mathrm{Z}} \mathrm{Y}_{\mathrm{e}}(\mathrm{Z})}{\sum_{\mathrm{all}}^{\mathrm{Z}} \mathrm{Y}_{\mathrm{e}}(\mathrm{Z})+\sum_{\mathrm{all}}^{\mathrm{Z}} \mathrm{Y}_{0}(\mathrm{Z})}
$$

where EOZ - even-odd proton enhancement factor,

$Y_{e}(Z)$ - isotopic independent yield of even $Z$ products,

$Y_{0}(Z)$ - isotopic independent yield of odd $Z$ products.

A similar expression was used for evaluating the even-odd neutron enhancement factor EON.

In the case of low energy fissioning systems with enough yield data available, Eq. (2) gives the magnitude of EOZ or EON directly. For other systems, various charge distribution models may be used. We have used the $Z_{p}$-model of WAHL ${ }^{14}$ for such calculations. 


\section{Results and discussion}

Figure 1 shows average post-neutron-emission masses of fission products as a function of the mass of the fissioning nucleous for various low energy fission processes calculated by Eq. (1). Data for the reactor neutron fission of 232 Th are from our work. $^{7}$ The rest of the mass yields are from Refs $2,16-18$. It is observed that the mean mass of the heavy product group is nearly constant at about $A=140$ for fissioning nuclides from $A_{f}=230$ to 246 . The mean mass of the light product group shows a steady increase with $A_{f}$ until about $A_{f}=250$. Beyond $A_{f}=250$ the average

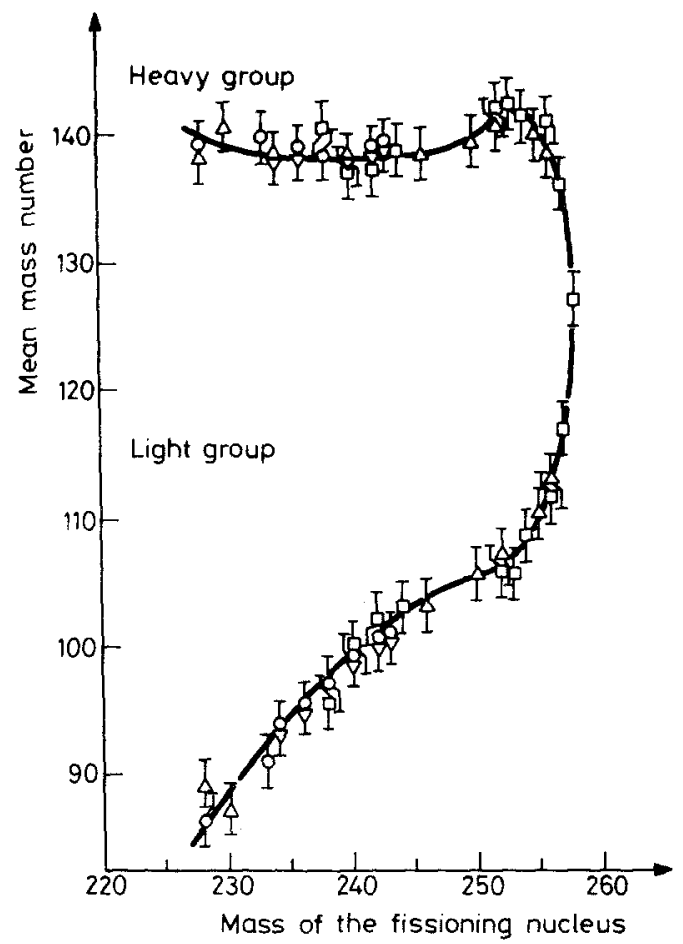

Fig. 1. Post-neutron-mission average masses as a function of mass of the fissioning nucleus: $\Delta$ thermal neutron induced fission, 0 reactor neutron induced fission, $\square$ spontaneous fission

mass of the heavy group first rises, whereas that of the light group somewhat flattens; afterwards the two groups rapidly converge and at $A_{f}=258$ we have symmetric fission. It is believed that the mass asymmetry in low energy fission, which turns into symmetric division with increasing $A_{f}$, is influenced by the existence of $Z=50$ and 
$\mathrm{N}=82$ proton and neutron closed shells in the heavy product group. However, it is not clear why the average heavy group mass stays constant at $A=140$ and not at the double magic $\mathrm{A}=132$. The available nuclear models cannot fully explain the observed mass divisions in low energy fission and this still remains one of the mysteries of the fission process.

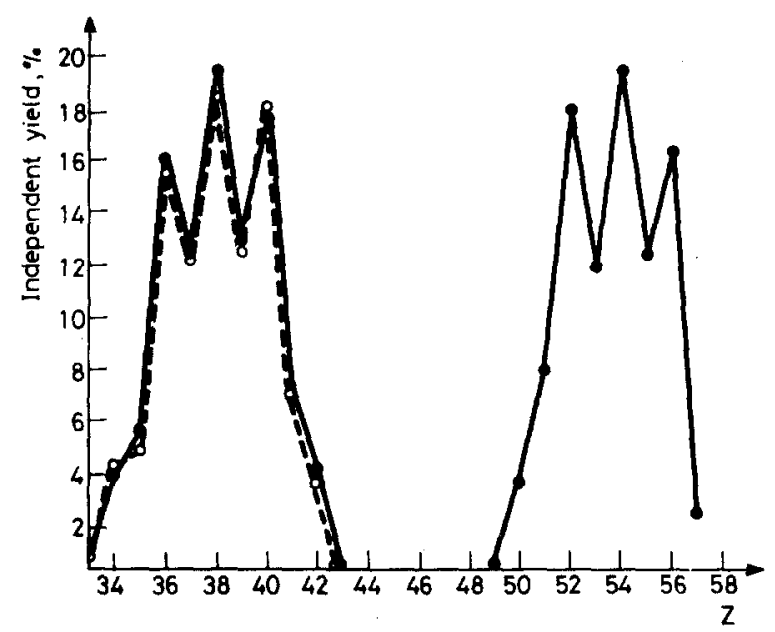

Fig. 2. Elemental yield distribution in the thermal neutron fission of ${ }^{235} \mathrm{U}: \bullet$ radiochemical yields based on CHUNG's data, ${ }^{10} \circ$ LOHENGRIN data of CLERC et al. ${ }^{12}$

The independent yields of individual elements formed in the thermal neutron fission of ${ }^{235} \mathrm{U}$ are shown in Fig. 2. The yield data of WAHL, ${ }^{2} \mathrm{CHUNG}^{10}$ and CLERC et al. ${ }^{12}$ have been used. The first two are based on radiochemical measurements, whereas the third used fission product recoil techniques. The results of the two independent type of measurements agree quite well although radiochemical measurements represent yields integrated over all kinetic energies of the products, whereas the LOHENGRIN measurements were made at the most probable kinetic energies. The charge asymmetry is very evident. It is interesting to observe that the distribution is narrow and only five elements in each of the light and heavy product groups are formed prominently; krypton to zirconium and tellurium to barium. Furthermore, yields of the elements of even atomic numbers are enhanced relative to those with odd numbers.

The isotonic yields of the light product group based on data of CLERC et al. ${ }^{12}$ are shown in Fig. 3. The distribution is much wider than the corresponding isotopic distribution and the even-odd neutron effect, EON is much smaller than the EOZ. Both results are probably due to the neutron evaporation process. 


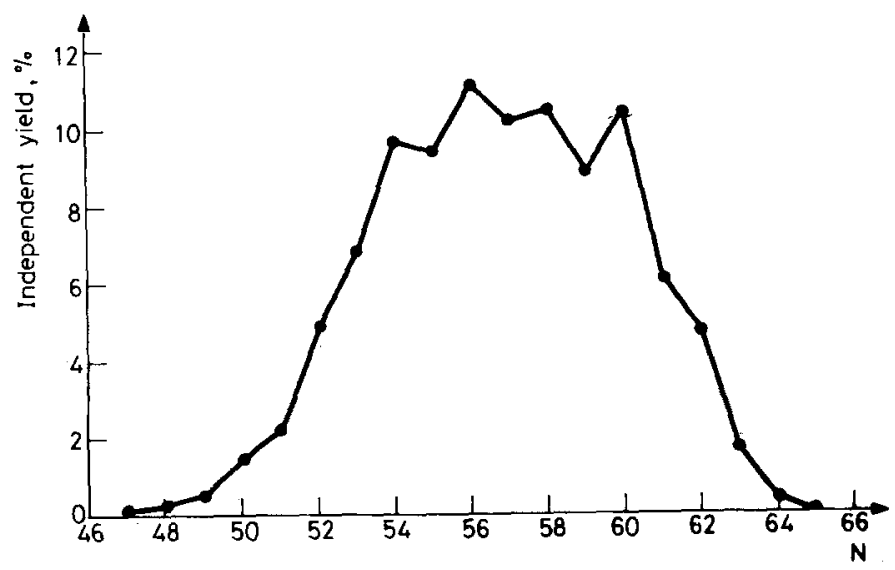

Fig. 3. Isotonic yield distribution in the light group products in thermal neutron fission of ${ }^{235} \mathrm{U}$, based on the data of CLERC et al. ${ }^{12}$

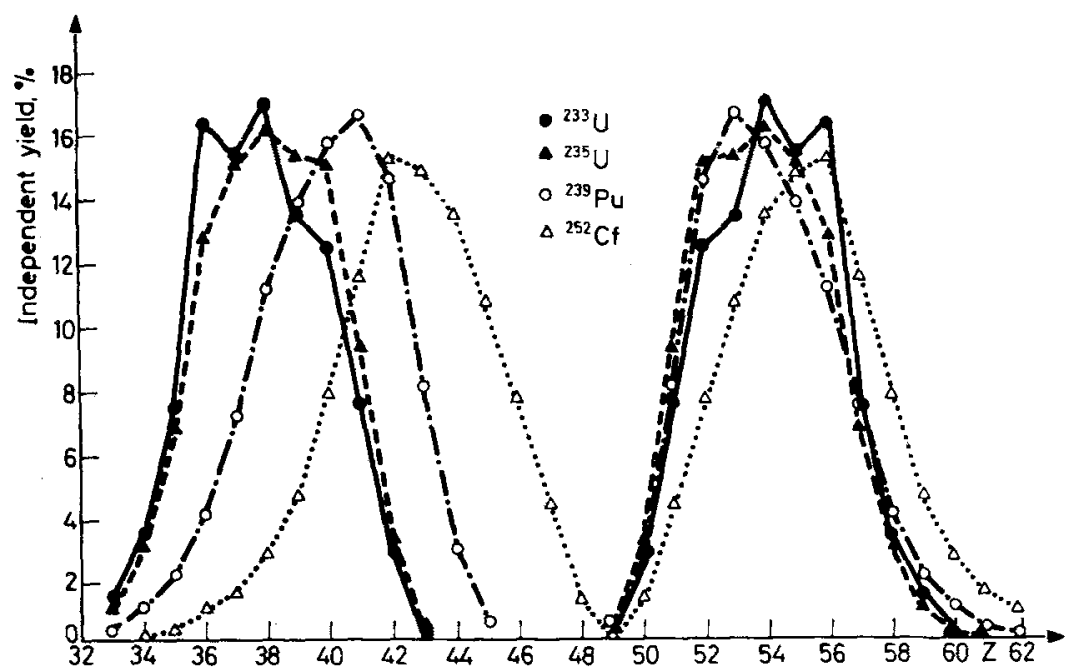

Fig. 4. Elemental yield distribution in the thermal neutron fission of ${ }^{235} \mathrm{U},{ }^{23}{ }^{3} \mathrm{U},{ }^{239} \mathrm{Pu}$ and the spontaneous fission of ${ }^{252} \mathrm{Cf}$ based on the X-ray measurements of REISDORF et al. ${ }^{19}$

REISDORF et al. ${ }^{19}$ carried out an extensive study of nuclear charge distribution in the thermal neutron fission of ${ }^{235} \mathrm{U},{ }^{233} \mathrm{U},{ }^{239} \mathrm{Pu}$ and spontaneous fission of ${ }^{252} \mathrm{Cf}$ by measuring $\mathrm{K} \mathrm{X}$-ray yields of the products. Figure 4 is based on their results. It is seen that the most probable charge of the heavy product group is constant, 


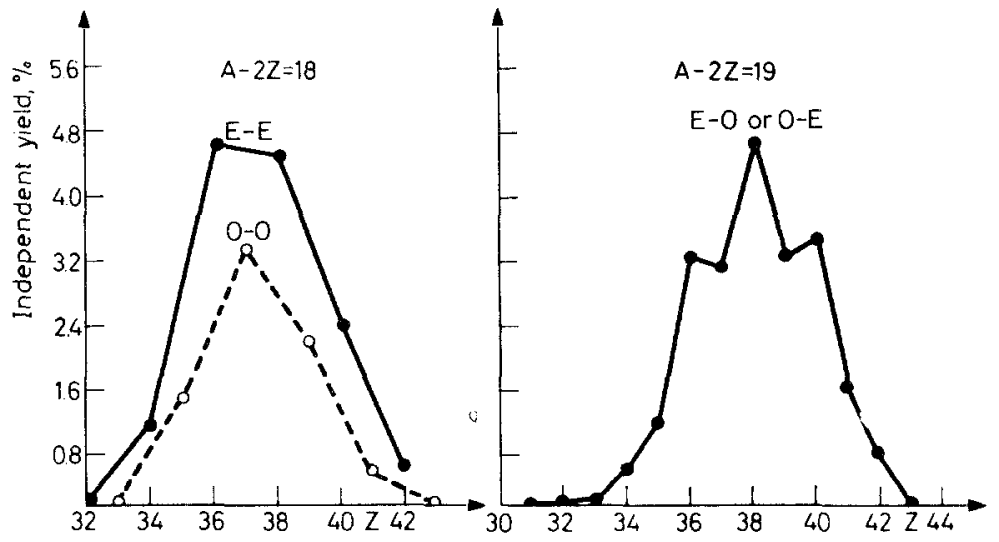

Fig. 5. Independent yield of products with an even $(E)$ or odd $(O)$ number of protons and neutrons in the thermal neutron fission of ${ }^{235} \mathrm{U}$ for the light mass peak. Curves are for constant excess neutrons of products with most available fission yields

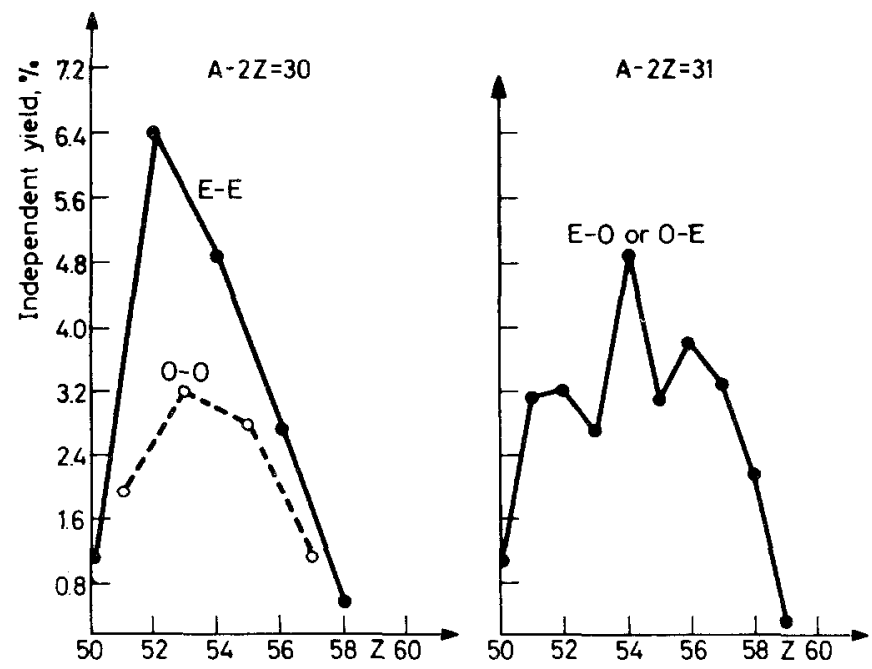

Fig. 6. independent yields of products with an even (E) or odd (O) number of protons and neutrons in the thermal neutron fission of ${ }^{235} \mathrm{U}$ for the heavy mass peak. Curves are for constant excess neutrons of products with most available fission yiclds

whereas that of the light group moves towards the heavy group with increasing $A_{f}$. This behavior is similar to that of the average product masses as shown in Figure 1. Another important observation from Figure 4 is that, with the possible exception of ${ }^{233} \mathrm{U}$, no significant even-odd effects seem to exist.

An alternative way of illustrating and estimating EOZ or EON effects is shown in Figs 5 and 6 . Here, independent yields of products with the same neutron excess 
values are plotted as a function of atomic number for light and heavy peaks respectively. The difference between even $Z$, even $N(E E)$ and odd $Z$, odd $N(O O)$ yields are striking. Furthermore, in the heavy peak the possible effects of $Z=50$ $\mathrm{N}=82$ shells make the EE curve much higher than the $\mathrm{OO}$ curve. The EO and $\mathrm{OE}$ curves clearly reveal that the proton nairing effect is much higher than that of neutron pairing.

A summary of yield data and the results of EOZ and EON calculations in the thermal neutron fission of ${ }^{235} \mathrm{U},{ }^{233} \mathrm{U},{ }^{239} \mathrm{Pu}$ and spontaneous fission of ${ }^{252} \mathrm{Cf}$ by Eq. (2) are given in Table 1. The yield data are almost complete only in the case

Table 1

Summary of independent yield data and results of EOZ, EON and specific yield calculations in the thermal neutron fission of ${ }^{35} \mathrm{U},{ }^{23} \mathrm{U}$, ${ }^{239} \mathrm{Pu}$ and sportaneous fission of ${ }^{252} \mathrm{Cf}$

\begin{tabular}{|c|c|c|c|c|}
\hline Parameter examined & $235 \mathrm{U}$ & ${ }^{233} \mathrm{U}$ & ${ }^{239} \mathrm{Pu}$ & ${ }^{252} \mathrm{Cf}$ \\
\hline \multicolumn{5}{|l|}{ Number } \\
\hline Even $\mathrm{Z}$ products & 103 & 84 & 73 & 37 \\
\hline Odd $Z$ products & 121 & 90 & 84 & 21 \\
\hline Even $N$ products & 99 & 88 & 74 & 47 \\
\hline Odd $\mathbf{N}$ products & 125 & 86 & 83 & 11 \\
\hline \multicolumn{5}{|l|}{ Yield } \\
\hline Even $Z$ products & 117.74 & 89.72 & 82.23 & 43.15 \\
\hline Odd $\mathrm{Z}$ products & 73.15 & 60.43 & 69.30 & 28.89 \\
\hline Even $\mathrm{N}$ products & 98.92 & 72.80 & 76.48 & 61.04 \\
\hline Odd N products & 91.97 & 79.35 & 75.05 & 11.00 \\
\hline $\mathrm{EOZ}$ & 1.23 & 1.20 & 1.08 & - \\
\hline EON & 1.04 & 0.96 & 1.01 & - \\
\hline \multicolumn{5}{|c|}{ Specific yield: $(\Sigma$ Yield $/ \Sigma$ Nuclide $)$} \\
\hline Even $\mathrm{Z}$ products & 1.14 & 1.07 & 1.13 & - \\
\hline Odd $\mathrm{Z}$ products & 0.60 & 0.67 & 0.83 & - \\
\hline Even $N$ products & 1.00 & 0.83 & 1.03 & - \\
\hline Odd $\mathrm{N}$ products & 0.73 & 0.92 & 0.90 & - \\
\hline$(\mathrm{EOZ})_{\mathrm{sp}}$ & 1.31 & 1.23 & 1.15 & - \\
\hline$(\mathrm{EON})_{\mathrm{sp}}$ & 1.16 & 0.95 & 1.07 & - \\
\hline \multicolumn{5}{|l|}{ Total yield } \\
\hline Light peak & 101.57 & 98.74 & 95.39 & 32.40 \\
\hline Heavy peak & 89.32 & 51.41 & 56.14 & 39.65 \\
\hline Total & 190.89 & 150.15 & 151.53 & 72.05 \\
\hline
\end{tabular}


of ${ }^{235} \mathrm{U}$. Here, there is about $10 \%$ independent yield yet to be measured in the heavy mass peak. In the case of ${ }^{233} \mathrm{U}$ and ${ }^{239} \mathrm{Pu}$ fission the data of the light mass peak region are almost complete, whereas about $50 \%$ of yields of the heavy peak are still lacking. For ${ }^{252} \mathrm{Cf}$, which is the next most studied fission process, most of the experimental independent yield data is still missing. Thus for ${ }^{252} \mathrm{Cf}$ and all the rest

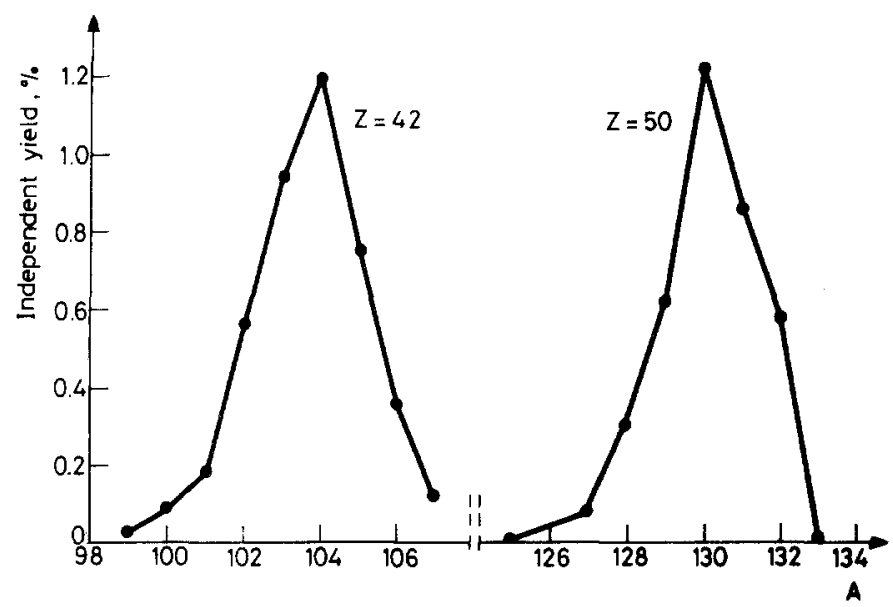

Fig. 7. Independent yield distribution of $Z=50$ and its complementary $Z=42$ isotopes in the thermal neutron fission of ${ }^{235} \mathrm{U}$

of the low energy fissioning nuclides, no meaningful analysis of EOZ and EON effects can be made using Eq. (2).

It is interesting that the number of even $\mathrm{Z}$ and even $\mathrm{N}$ products with measured yields are appreciably less than the corresponding odd ones (Table 1). On the basis of the pairing effect in nuclear structure, one would expect the opposite to be true, as in the case for example of the number of stable even and odd isotopes found in natu e, 212 versus 54 , respectively. This reversal of the expected behavior is probably caused by the $\mathrm{Z}=50, \mathrm{~N}=50$ and $\mathrm{N}=82$ nuclear shells. The yields of products in the vicinity of shells, are enhanced, while the number of products formed is suppressed because of preferential formation. Figures 7 and 8 illustrate the influence of nuclear shells on the independent yields in the fission of ${ }^{235} \mathrm{U}$. The effect of the $Z=50$ proton shell and its complementary $Z=42$ is shown in Fig. 7. The yield distribution is narrow and symmetric. The corresponding influence of $N=50$ and $\mathrm{N}=82$ neutron shells is shown in Figure 8. The enhancement of yields and narrow symmetric distribution of $\mathrm{N}=82$ isotones is apparent. The $\mathrm{N}=50$ effect is not as pronounced. 


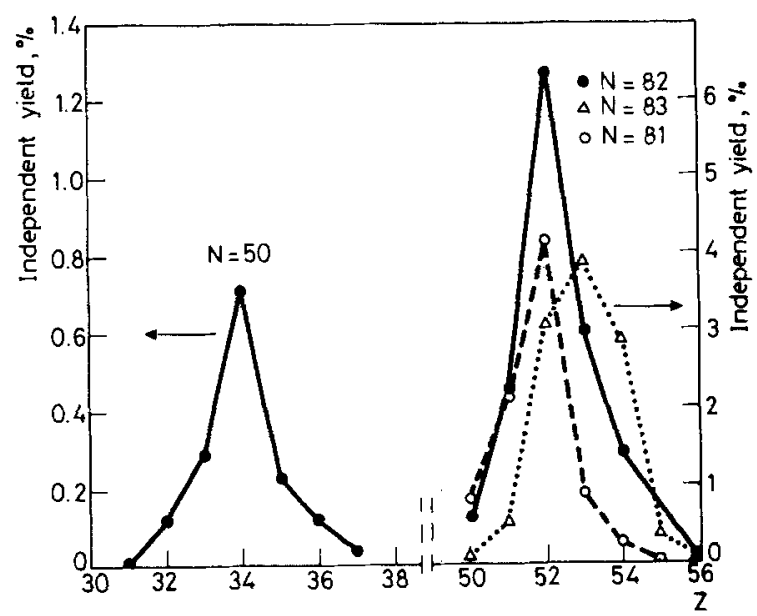

Fig. 8. Independent yield distribution of $N=50$ and $N=81,82,83$ isotopes in the termal neutron fission of ${ }^{235} \mathrm{U}$

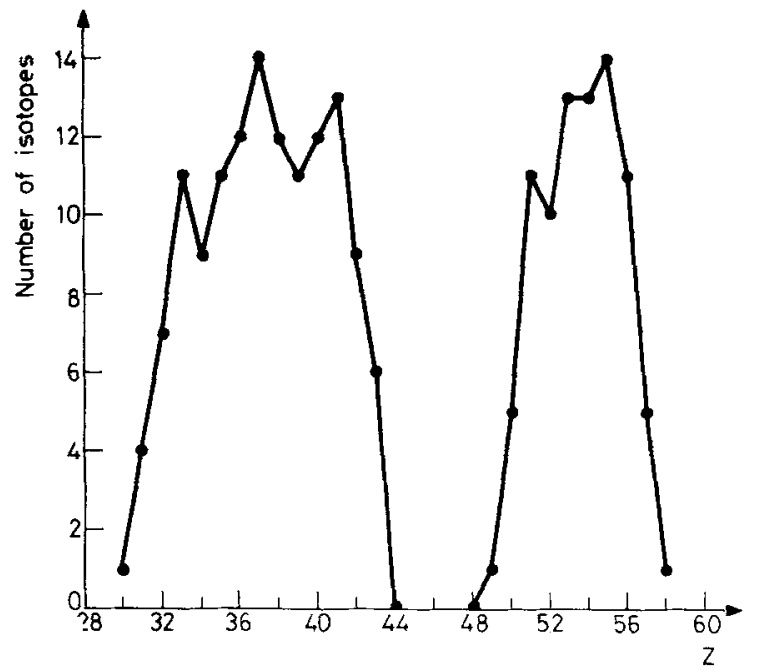

Fig. 9. Distribution of the number of isotopes formed as a function of atomic number in the thermal neutron fission of ${ }^{235} \mathrm{U}$

Figure 9 shows the distribution of the number of isotopes formed in the fission of ${ }^{235} \mathrm{U}$ as a function of atomic number. Since the experimental measurement of independent yields is almost complete for ${ }^{235} \mathrm{U}$ fission, we assume that all the products with reasonably independent yields are included in this comparison. The striking feature of the isotopic distribution is the existence of fine structure peaks with 


\section{H. N. ERTEN, N. K. ARAS: FINE STRUCTURE OF MASS AND CHARGE}

somewhat more prominence in the light peak region. The heavy region is considerably narrower, a clear effect of $\mathrm{Z}=50, \mathrm{~N}=82$ shells. A comparison of this figure with the elemental yield distribution given in Figure 2 points to some similarities, namely, fine structure effects and also important differences. The number of odd $\mathrm{Z}$ isotopes are enhanced relative to even $Z$ ones, an effect just the opposite of the yield enhancements of Figure 2. As was pointed above, this observed reversal is due to the influence of nuclear shells. Thus it appears that what is calculated as EOZ or EON from Eq. (2), also contains contributions from nuclear shells effects. From such considerations we have defined and computed specific yield per nuclide ( $\Sigma$ Yield $/ \Sigma$ Nuclide) values for products with even and odd numbers of protons and neutrons as shown in Table 1. It is seen that the specific yields of even $Z$ products are significantly above unity and those of odd $Z$ products considerably below unity.

The results of even-odd yield enhancement factor calculations EOZ and EON using Eq. (2), in the thermal neutron fission of ${ }^{235} \mathrm{U},{ }^{233} \mathrm{U}$ and ${ }^{239} \mathrm{Pu}$ are given in Table 1. The available yield data of ${ }^{252} \mathrm{Cf}$ fission were not sufficient for such treatment. The EOZ values are pronounced only for ${ }^{233} \mathrm{U}$ and ${ }^{235} \mathrm{U}$ fission, whereas the EON values are very close to unity, indicating no effect. On the other hand, if the specific yields defined above are used in Eq. (2), the even-odd enhancement factors, $(\mathrm{EOZ})_{\mathrm{sp}}$ and $(\mathrm{EON})_{\mathrm{sp}}$ come out somewhat higher than those using independent yields (Table 1). The increased values may be attributed to the influence of nuclear shells. From such calculations we estimate about $7 \%$ yield enhancement due to shell effects, the rest being due to true pairing. This estimated magnitude of the shell effect contribution to the even-odd effect is obtained considering all the fission products in all regions; a sort of average contribution. If one takes into account, however, that the shell effects are most pronounced at the shell regions and their complementaries only, then the magnitude of the shell effects should increase considerably within these regions and would be negligible outside. These findings are in line with those of CLERC et al. ${ }^{\mathbf{2}}$ who found shell effect modulations in EOZ and EON values for the light peak products and the observations of WAHL ${ }^{20}$ in the symmetric region where no even-odd and/or shell effects were found.

The above treatment is only possible with a complete set of yield data and since this is lacking for most of the low energy fissioning systems, one may use various models of charge distribution to estimate the even-odd effect. The $Z_{p}$ model of $\mathrm{WAHL}^{2}$ is the most widely accepted and used model for this purpose. Using the available experimental yield data and the method of least squares, one obtains parameters of charge distribution for individual isobaric mass chains as well as for the whole fissioning nuclide. We have carried out such charge distribution calculations using the $\mathrm{Z}_{\mathrm{p}}$-model for several low energy fissioning systems. The even-odd proton and neutron enhancement factor results are shown in Fig. 10. The EOZ effect is well 
established and prominent in ${ }^{235} \mathrm{U}$ and ${ }^{233} \mathrm{U}$ fissions only. In the fission of ${ }^{232} \mathrm{Th}$, ${ }^{239} \mathrm{Pu},{ }^{245} \mathrm{Cm}$ and ${ }^{249} \mathrm{Cf}$ the EOZ effect is of the order of the estimated shell effect. In the rest of the fissioning systems it is not significantly different from 1.0. The EON effects are found to be small in all cases and somewhat larger than 1.0 for ${ }^{233} \mathrm{U}$ and ${ }^{235} \mathrm{U}$ fissions only.
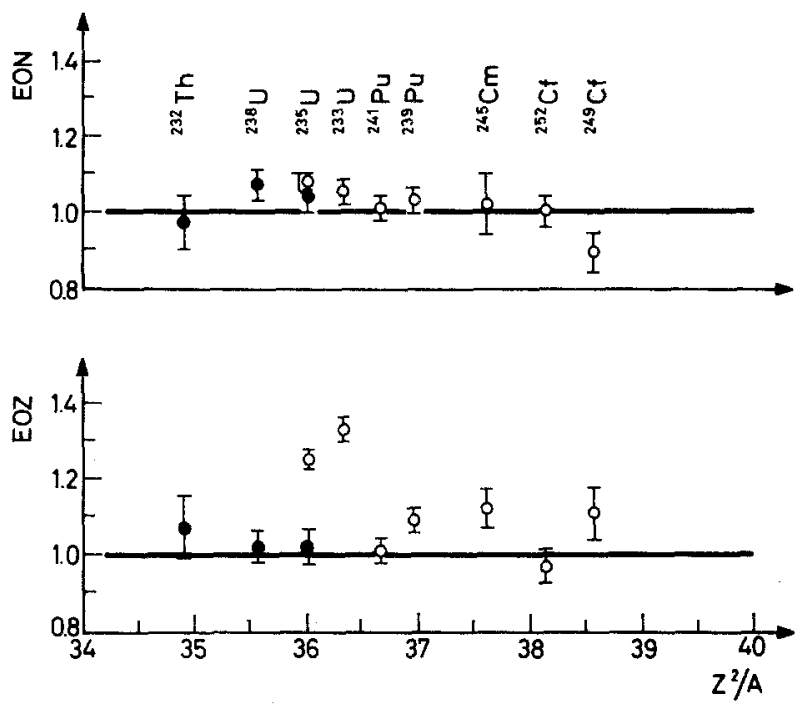

Fig. 10. Comparison of EOZ and EON values for various low energy fission processes calculated according to the $Z_{\mathrm{p} \text {-model of WAHL. }}{ }^{2}$ The experimental yields used in the calculations are from References; $;, 10_{0} 15,21-24$ fast neutron fission, 0 thermal neutron and spontaneous fission

A comparison with the results of Table 1 show that model calculations generally overestimate the even-odd effects. In the most extreme case of ${ }^{233} \mathrm{U}$ this overestimation is about $10 \%$. These findings suggest that the even-odd effects in low energy fission should be taken into account with caution and such corrections should not be applied to all products. Apart from ${ }^{235} \mathrm{U}$ and possibly ${ }^{233} \mathrm{U}$ and ${ }^{239} \mathrm{Pu}$ fission, experimental data to enable the type of direct analysis as given in Table 1 are lacking.

More experimental yield measurements and model calculations are certainly needed in order to clarify, understand and quantify these important fine structure effects in low energy fission. 


\section{References}

1. O. HAHN, F. STRASSMANN, Z. Naturwiss., 27 (1939) 11.

2. A. C. WAHL, Atomic Data and Nuclear Data Tables, Vol. 39, 1988.

3. C. D. COR YELL, M. KAPLAN, R. D. FINK, Can. J. Chem., 39 (1951) 646.

4. H. GÖKTÜRK, O. BIRGUUL, H. N. ERTEN, N. K. ARAS, J. Inorg. Nucl. Chem., 37 (1975) 2247.

5. H. N. ERTEN, O. BIRGUL, N. K. ARAS, J. Inorg. Nucl. Chem., 40 (1978) 183.

6. H. N. ERTEN, N. K. ARAS, J. Inorg. Nucl. Chem., 41 (1979) 149.

7. H. N. ERTEN, A. GRUTTER, E. RÖSSLER, H. R. VON GUNTEN, Nucl. Sci. Eng., 79 (1981) 167.

8. H. N. ERTEN, A. GRUTTTER, E. RÖSSLER, H. R. VON GUNTEN, Phys. Rev. C., 25 (1982) 2519.

9. L. TOPPARE, H. N. ERTEN, N. K. ARAS, Can. J. Chem., 61 (1983) 649.

10. C. CHUNG, Radiochim. Acta., 39 (1986) 113.

11. S. AMIEL, H. FELDSTEIN, Phys. Rev. C., 11 (1975) 845.

12. H. G. CLERC, W. LANG, H. WOHLFARTH, K. H. SCHMIDT, H. SCHRADER, K. E. PHERDEKAMPER, R. JUNGMAN, Z. Phys., A274 (1975) 203.

13. G. MARIOLOPOULOS, Ch. HAMELIN, J. BLACHOT, J. P. BOCQUET, R. BRISSOT, J. CRANSON, H. NIFENECKER, Ch. RISTORI, Nucl, Phys. A361 (1981) 213.

14.D. R. SAROHA, R. K. GUPTA, Phys. Rev. C., 29 (1984) 1101.

15. A. C. WAHL, J. Radioanal. Chem., 55 (1980) 111.

16. B. F. RIDER, NEDO-12154, 1980.

17. D. C. HOFFMAN, M. M. HOFFMAN, Ann. Rev. Nucl. Sci., 24 (1974) 24.

18. D. C. HOFFMAN, Phy sics and Chemistry of Fission, Vol. 2, IAEA, Vienna, 1980, p. 275.

19. W. REISDORF, J. P. UNIK, H. C. GRIFFIN, L. E. GLENDENIN, Nucl. Phys. A, 177 (1971) 337.

20. A. C. WAHL, Phys. Rev, C, 32 (1985) 184.

21. R. BRISSOT, J. CANSON, Ch. RISTORI, J. BOCOUET, A. MOUSSA, Nucl. Phys. A, 282 (1977) 109.

22. H. R. VON GUNTEN, K. F. FLYNN, L. E. GLENDENIN, Phys. Rev., 161 (1967) 1192.

23. J. K. DICKENS, J. W. CONNELL, Phys. Rev. C, 23 (1981) 331.

24. H. H. MAIXLER, Ph D. Tesis, Nuclear Chemistry Institute, University of Mainz, 1980. 